\title{
Characterization and Synthesis of Some One-dimensional Heterojunctions
}

Jing Zhu , Jun Luo and Kuiqing Peng

Electron Microscope Laboratory, School of Materials Science and Engineering, Tsinghua University, Beijing 100084, P.R.China

In the recent few years, some one-dimensional (1D) heterostructures, such as Si-CNT (carbon nanotube $)^{[1]}, \mathrm{Si}_{-} \mathrm{SiGe}^{[2]}, \mathrm{InAs}_{-} \mathrm{InP}^{[3]}, \mathrm{GaAs}-\mathrm{GaP}^{[4]}$ and so on, have been paid much attention to because heterostructures are widely used in many miniature devices.

By using a doable combination of electrochemical deposition and chemical vapor deposition, we have synthesized successfully some 1D heterojunctions, such as $\mathrm{Ni}_{-} \mathrm{CNT}^{[5]}, \mathrm{Ag}^{-\mathrm{Si}^{[5]}}$ and $\mathrm{Pt}_{6} \mathrm{Si}_{5}-\mathrm{Si}^{[6]}$, shown in Fig.1. Their diameters are controlled by the sizes of the pores of the templates used in the preparations.

By using a planar silicon p-n junction as the precursor in a self-assembling nanoelectrochemical technique $^{[7]}$, large-area silicon nanowire (SiNW) p-n diode arrays have been successfully prepared, shown in Fig.2a. The method is rather simple. After a treatment of the planar p-n junction in an aqueous HF solution containing silver nitrate, the SiNW p-n diode arrays can be obtained on silicon substrates at near room temperature. Fig. $2 \mathrm{~b}$ shows a typical I-V curve for a bundle of SiNW p-n diodes. The I-V curve reveals the rectifying behavior similar to that of planar silicon $\mathrm{p}-\mathrm{n}$ junctions. These $1 \mathrm{D}$ heterojunctions are believed significant for the development of nano-devices.

\section{References}

[1] JT Hu, OY Min, PD Yang, et al. Controlled growth and electrical properties of heterojunctions of carbon nanotubes and silicon nanowires. Nature, 1999, 399(6731): 48.

[2] YY Wu, R Fan, PD Yang. Block-by-Block Growth of Single-Crystalline Si/SiGe Superlattice Nanowires. Nano Letters, 2002, 2(2): 83.

[3] MT Bjork, BJ Ohlsson, T Sass, et al. One-dimensional Steeplechase for Electrons Realized. Nano Letters, 2002, 2(2): 87.

[4] MS Gudiksen, LJ Lauhon, J Wang, et al. Growth of nanowire superlattice structures for nanoscale photonics and electronics. Nature, 2002, 415(6872): 617.

[5] J Luo, L Zhang, YJ Zhang, et al. Controlled growth of one-dimensional metal-semiconductor and metal-carbon nanotube heterojunctions. Advanced Materials, 2002, 14(19): 1413. 
[6] J Luo, L Zhang, J Zhu. Novel synthesis of $\mathrm{Pt}_{6} \mathrm{Si}_{5}$ nanowires and $\mathrm{Pt}_{6} \mathrm{Si}_{5}-\mathrm{Si}_{\text {nanowire }}$ heterojunctions by using polycrystalline Pt nanowires as templates. Advanced Materials, 2003, in press.

[7] KQ Peng, YJ Yan, SP Gao, J Zhu. Synthesis of large-area silicon nanowire arrays via self-assembling nanoelectrochemistry. Advanced Materials, 2002, 14(16): 1164.
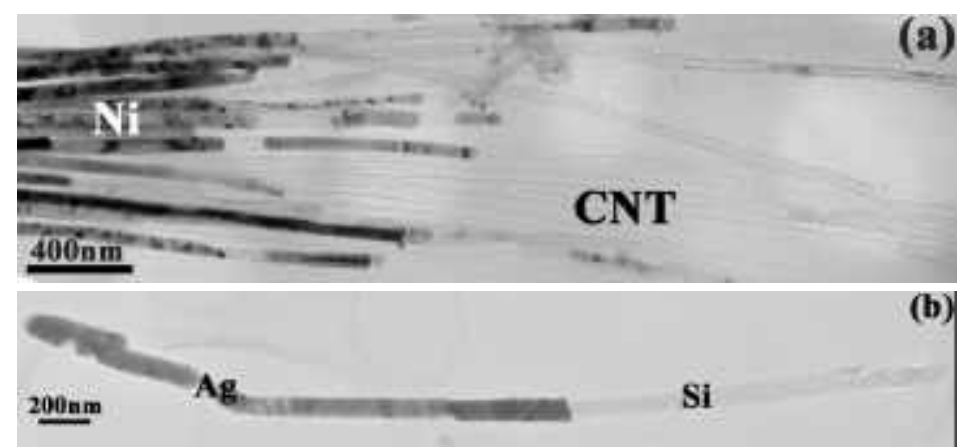

(b)

(c)
Fig. 1 Transmission electron microscope (TEM) images of (a) Ni-CNT, (b) Ag-Si and (c) $\mathrm{Pt}_{6} \mathrm{Si}_{5}-\mathrm{Si}$ $1 \mathrm{D}$ heterojunctions.
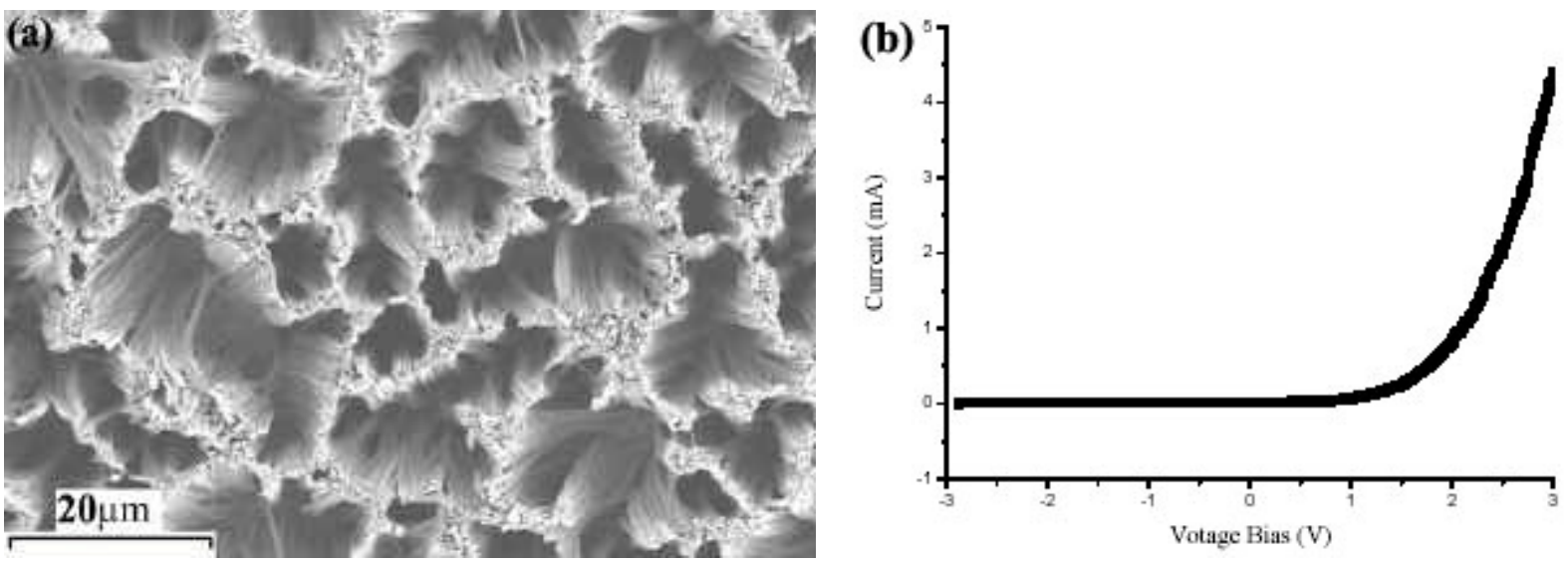

Fig. 2 (a) A scanning electron microscope (SEM) image of SiNW p-n diode arrays. (B) An I-V curve of a bundle of SiNW p-n diodes. 